\title{
A Multiclass, Multimodal Dynamic Traffic Assignment Model with Departure Time
}

\author{
Meng Meng, ${ }^{1,2}$ Chunfu Shao, ${ }^{1}$ Yiik Diew Wong, ${ }^{2}$ and Jie Zhang ${ }^{2}$ \\ ${ }^{1}$ MOE Key Laboratory for Urban Transportation Complex Systems Theory and Technology, Beijing Jiaotong University, \\ Beijing 100044, China \\ ${ }^{2}$ Centre for Infrastructure Systems, School of Civil and Environmental Engineering, Nanyang Technological University, \\ Singapore 639798
}

Correspondence should be addressed to Chunfu Shao; cfshao@bjtu.edu.cn

Received 5 April 2014; Revised 30 June 2014; Accepted 30 June 2014; Published 14 July 2014

Academic Editor: Paolo Lonetti

Copyright (C) 2014 Meng Meng et al. This is an open access article distributed under the Creative Commons Attribution License, which permits unrestricted use, distribution, and reproduction in any medium, provided the original work is properly cited.

\begin{abstract}
The paper develops a multiclass, multimodal dynamic traffic equilibrium model with consideration of the departure time choice problem. Travelers choose the departure time and the route simultaneously with a Logit-based structure. The route travel cost is a summation of travel time and schedule delay which is associated with arrival time at destination. In addition, the travelers are classified into three groups according to their value of time. A variational inequality (VI) formulation is proposed based on the equilibrium conditions. Two examples are given to testify the effectiveness of the model and the solution algorithm. The model can give the optimal travel route as well as the best departure time, which would contribute to traffic control and dynamic route guidance.
\end{abstract}

\section{Introduction}

Dynamic traffic assignment (DTA) models which explicitly considered time varying flows in the traffic assignment were first systematically formulated into analytical formulation by Merchant and Nemhauser [1]. As dependent on the assumptions about the route choice criterion, DTA models can be grouped into two categories: deterministic dynamic traffic assignment (DDTA) models and stochastic dynamic traffic assignment (SDTA) models [2]. DDTA models assume that all travelers have perfect information of network condition and they will make their best choice without any errors which is found to be unrealistic in practice. SDTA models relax these assumptions and consider that travelers will choose their perceived best route based on their individualistic understanding about the network condition. Since the SDTA models are more credible, the idea was much developed in the literature and in many cases researchers provided strong mathematical properties, as discussed elsewhere [3-5].

Recently, there has been a significant interest in modeling the combined departure time and dynamic user equilibrium assignment (DDUE) problem in urban areas. DDUE models can be classified into three groups based on the applied technique: analytical models [6-10], simulation models [11], and cell models [12]. As the analytical models provide a theoretical foundation and lend support to results obtained from other models, it is always a popular topic in this field. Hendrickson and Kocur [13] described this problem systematically and pointed out that if the dynamic stochastic user equilibrium assignment (DSUE) and departure time problem are combined, then the cost, including that of travel plus penalties for timing of the journey, at which travel takes place has a single value for each origin-destination pair in a network. Ran and Boyce [14] and Chen [15] have established variational inequality (VI) models to discuss the DDUE problem, which require complex computing process as they include not only the link variable but also the OD demand variable. Lam and Huang [16] developed a path-based DDUE model with deterministic queue assumption. Lim and Heydecker [8] investigated a Logit-based combined departure time and dynamic stochastic user equilibrium assignment (DDSUE) model and showed the existence of DDSUE condition as well as explored its properties. It extended the DDUE problem into the stochastic case which is more realistic and has 
attracted much attention among researchers. Lam et al. [17] proposed a time-dependent network equilibrium model that simultaneously considers a traveler's choice of departure time, route, parking location, and parking duration in road networks with multiple user classes and multiple parking facilities. Li [18] presented a genetic algorithm for solving dynamic simultaneous route and departure time equilibrium problem. Not only can a flow-swapping process in the algorithm guarantee the flow conservation constraints between an $O D$ pair, but it also accelerates the convergence velocity of the algorithm. Lately, Meng et al. [19] also proposed a pathbased DDSUE model in a multimodal network.

Most traditional traffic assignment models assume that the travelers have the same value of time (VOT), namely, the homogeneity condition [20-23]. In fact, however, the transportation network has its heterogeneity as the travelers have different requirement for travel time due to the social status, economic income, and the nature of work. Multimodal network equilibrium models considering the multiclass travelers who have different VOT have not received much attention in the literature. Lu et al. [24] developed a bicriterion dynamic user equilibrium (BDUE) model, which aimed to capture users' path choices in response to time-varying toll charges and hence explicitly considers heterogeneous users with different VOT preferences in the underlying path choice decision framework. Zhang et al. [25] assumed that both travel time disutility and time-irrelevant travel disutility depend only on the particular link flow. The time-irrelevant travel disutility allows the consideration of other travel expense except travel time, for example, emission, noise, discomfort, and insecurity. They developed a multiclass multicriterion mixed equilibrium model, where drivers have multiple criteria in evaluating the travel disutility and follow either user equilibrium (UE) principle or Cournot-Nash (CN) principle. Wang and Huang [26] provided a theoretical investigation of the multicriterion traffic assignment, including the costversus-time network equilibrium and system optimum in a network with continuous VOT distribution and elastic demand.

This paper, in turn, focuses on the development of a new multiclass, multimodal network equilibrium model with departure time. The model has the following novel significant features.

(1) It extends the classical road network problem into the multimodal network case, including four traffic modes: car, subway, bus, and bicycle. Moreover, the combined-mode trips are considered in which travelers are allowed to transfer from one traffic mode to another one. Such extension would be the advanced step of the DDSUE problem towards the comprehensive transportation system and will contribute to travel guidance in ITS (intelligent transportation system) application.

(2) It investigates the multiclass travelers in the system according to different VOT. Specifically, the relation between daily volume of traffic and cost incurred from traveling is also investigated. Hence, the model has implications to different classes of travelers not

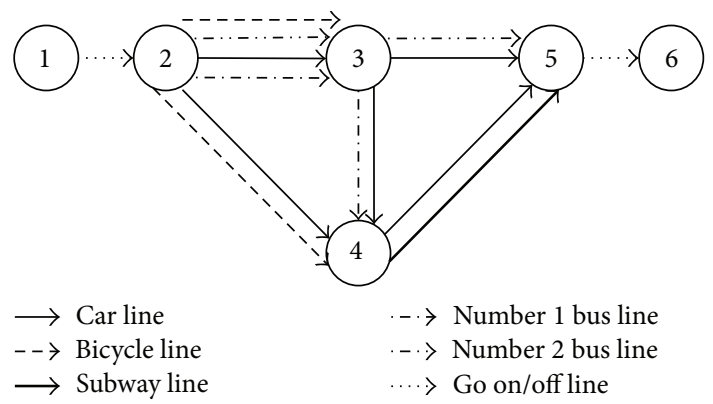

Figure 1: Multimodal transportation network.

only for the route choice, but also for the departure time choice.

This paper not only develops such a model, but also provides solution algorithm and application examples. The outline of the paper is as follows. The next section analyses the multimodal network equilibrium condition and the dynamic constraints. Section 3 develops the multiclass, multimodal dynamic traffic network equilibrium model with departure time. The algorithm which can be applied to solve the proposed model is given in Section 4. Numerical examples are provided in Section 5. The last section summarizes the conclusions and major findings.

\section{Multimodal Network Equilibrium Condition}

2.1. Multimodal Network. In this paper, the super network structure in $\mathrm{Wu}$ and Lam [27] is adopted to represent the multimodal transportation network. The super network is constructed such that the method for each mode is represented individually on separate layers that are interconnected by transfer links. This structure allows a complete representation of multimodal trips as well as the single mode trips. Moreover, the transfer behavior is well modeled by the interaction link. As an example, consider a simplified network shown in Figure 1. It consists of four traffic modes: car, subway, bus, and bicycle. Go on/off lines are virtual routes which help us to describe the origin and destination line. The corresponding representation using the super network structure is shown in Figure 2. The super network comprises the four subnetworks covering all the traffic modes, starting from node 1 and ending at node 17. The individual layers representing different traffic modes, in order, are car network, subway network, bicycle network, and bus network. The solid line denotes the driving/running line in each layer, while the dotted line is the transfer or on/off link.

Given a multimodal transportation network $G=(N, L)$, where $N$ is the set of node and $L$ is the set of link, $O$ is the set of origin node, $O \subset N, D$ is the set of destination node, $D \subset$ $N$, $o$ is an origin node, $o \subset O, d$ is a destination node, $d \subset D$, $P^{o d}$ is the set of path between $O D$ pair $o d$, each path $p$ is an available travel "mode-route" super path, $p \in P^{o d},\left[s_{0}, s_{1}\right]$ is the study period, $s, t \in\left[s_{0}, s_{1}\right], T^{o d}$ is the traffic demand between $O D$ pair $o d$, and $\theta_{t}$ and $\theta_{r}$ are the scale parameters, 


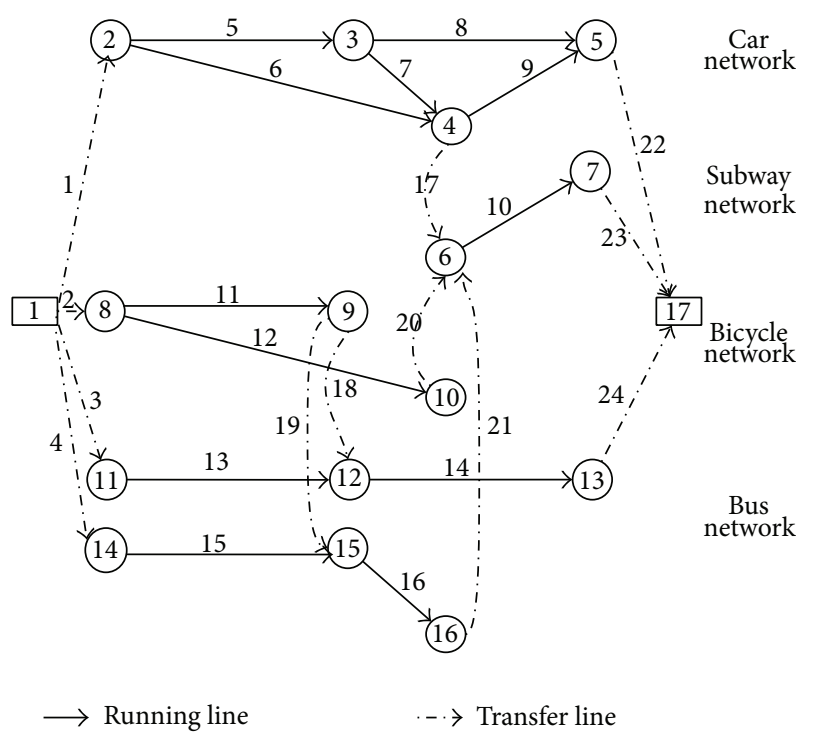

FIGURE 2: Super network.

where $\theta_{t}$ is a positive dispersion parameter for departure time choice and $\theta_{r}$ is a positive dispersion parameter that controls the assignment to routes of non-minimal cost.

2.2. Equilibrium Condition. In order to design the solution algorithm, the discrete mathematics method is used herein for analysis and formulation. Assume that study period $\left[s_{0}, s_{1}\right]$ is sufficiently long such that all the vehicles departing from the origin node in study period can leave the network in the study period. Divide the study period into $S$ numbers of small time interval with length of $\Delta=\left(s_{1}-s_{0}\right) / S$. The rule in the division is as follows: vehicle which drives into a segment in a time interval cannot leave this segment in the same time interval. The Logit model is introduced to describe the departure time preference as follows:

$$
e^{o d}(s)=T^{o d} \mu^{o d}(s)=T^{o d} \frac{\exp \left[-\theta_{t} c^{o d}(s)\right]}{\sum_{s=1}^{S} \exp \left[-\theta_{t} c^{o d}(s)\right]} \quad \forall s,
$$

where $e^{o d}(s)$ is the departure flow at time $s$ for $O D$ pair $o d$, $\mu^{o d}(s)$ is the temporal density of departures from $o$ to $d$ at time $s$, and $c^{o d}(s)$ is the typical departure cost incurred by a traveller that enters the network at time $s$ for $O D$ pair od. Based on the expected utility theory, $c^{\text {od }}(s)$ can be expressed as

$$
c^{o d}(s)=-\frac{1}{\theta_{r}} \ln \sum_{p \in P_{o d}} \exp \left[-\theta_{r} c_{p}(s)\right] \quad \forall s,
$$

where $c_{p}(s)$ is the cost of travel using path $p$ entering the network at time $s$, without regard to the influence of money cost, the travel cost on path $p$ normalized to unity including the path travel time $\tau_{p}(s)$ and the schedule delay $f^{d}(s)$ that is associated with arrival at the destination $d$ at time $s$ is given by

$$
c_{p}(s)=\tau_{p}(s)+f^{d}\left(s+\tau_{p}(s)\right) .
$$

The schedule delay in this paper is assumed to be continuous in time and follows the piecewise linear form as follows:

$$
f^{d}(s)= \begin{cases}(\bar{s}-\delta-s \Delta) m_{1}^{i} & s \Delta<\bar{s}-\delta \\ 0 & \bar{s}-\delta \leq s \Delta \leq \bar{s}+\delta \\ (s \Delta-\bar{s}-\delta) m_{2}^{i} & s \Delta>\bar{s}+\delta\end{cases}
$$

where $\bar{s}$ is the ideal arrival time, $\Delta$ is an indifference time, $i$ is the class of travelers, and $m_{1}^{i}$ and $m_{2}^{i}$ are, respectively, the unit penalties for early and late arrival. This kind of piecewise linear schedule delay has been adopted by many researchers including Vickrey [28], Hendrickson and Kocur [13], and Arnott et al. [29] and satisfies the requirement of the present analysis of being continuous in time.

Let the initial departing flow from $o$ to $d$ be $e_{1}^{o d}(s)=$ $e^{\text {od }}(1)$; then

$$
e^{o d}(1)=T^{o d} \mu^{o d}(1)=T^{o d} \frac{\exp \left[-\theta_{t} c^{o d}(1)\right]}{\sum_{s=1}^{S} \exp \left[-\theta_{t} c^{o d}(s)\right]} .
$$

Substitution (5) into (1) and rearranging,

$$
e^{o d}(s)=\exp \left\{-\theta_{t}\left[c^{o d}(s)-c^{o d}(1)\right]\right\} e^{o d}(1) \quad \forall s .
$$

The Logit model is introduced to describe the route choice preference as follows:

$$
e_{p}(s)=e^{o d}(s) \mu_{p}(s)=e^{o d}(s) \frac{\exp \left[-\theta_{r} c_{p}(s)\right]}{\sum_{p \in P_{o d}} \exp \left[-\theta_{r} c_{p}(s)\right]} \quad \forall s,
$$

where $e_{p}(s)$ is the inflow to path $p$ at the time $s, \mu_{p}(s)$ is the proportion of those travelers at time $s$ that enter path $p$. Based on the tree choice theory, $\theta_{r} \geq \theta_{t}>0$.

Above all, we can conclude that the dynamic departure time/stochastic user equilibrium condition in a multimodal network can be described as follows: in equilibrium state of a multimodal transportation network, no traveler can improve his perceived travel cost by unilaterally changing his departure time and modal-route combination; the departure time and modal route choices are calculated by (1) and (7).

\subsection{Dynamic Constraint Condition}

2.3.1. Link State Equations. In the dynamic traffic assignment problem, traffic load is used to represent the traffic state on the link. The basic formulation of discrete link state equation is

$$
x_{a}(s)-x_{a}(s-1)=\left(e_{a}(s)-g_{a}(s)\right) \Delta, \quad \forall s, a,
$$

where $e_{a}(s)$ is the inflow to link $a$ at time $s, g_{a}(s)$ is the exit flow from link $a$ at time $s$, and $x_{a}(s)$ is the number of vehicles in the queue on link $a$ at time $s$. Equation (8) expresses the marginal changes of vehicle numbers on link $a$ at time $s$ that is equal to the difference value between inflow and exit flows on link $a$ at time $s$. 


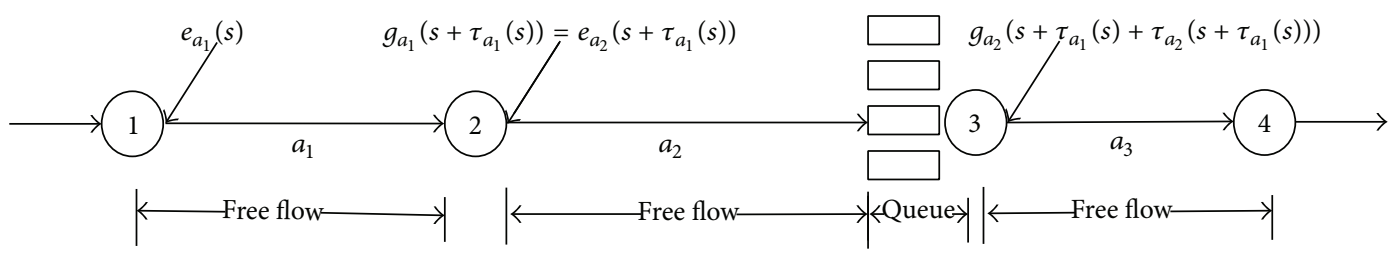

FIgURE 3: Path point-queue model.

Based on the super network theory, we extend (8) to the super path level as follows:

$$
x_{p}(s)-x_{p}(s-1)=\left(e_{p}(s)-g_{p}(s)\right) \Delta, \quad \forall s, a,
$$

where $e_{p}(s)$ is the inflow to path $p$ at time $s$ and $g_{p}(s)$ is the exit flow from path $p$ at time s. $x_{p}(s)$ is the number of vehicles on path $p$. Equation (9) expresses the marginal change of vehicles on path $p$ at time $s$ that is equal to the difference value between inflow and exit flows on path $p$ at time $s$.

2.3.2. Travel Time Function. Following the results of Lim and Heydecker [8], we use a deterministic queuing model, which depends only on the amount of traffic on that link. In the deterministic queuing model, the link constitutes two parts: free-flow part and queue part at the exit. Travel time on a link can be expressed as

$$
\tau_{a}(s)=\Phi_{a}+\frac{x_{a}(s)}{Q_{a}} \quad \forall a, s,
$$

where $\tau_{a}(s)$ is the travel time on link $a$ and $x_{a}(s)$ is the number of vehicles in the queue on link $a$ at time $s$. For the driving link, $\Phi_{a}$ is the free-flow travel time on link $a$ and $Q_{a}$ is the traffic capacity of link $a$; for the transfer link, $\Phi_{a}$ is the travel time on link $a$ including the walking time and waiting time and $Q_{a}$ is a parameter reflecting the transfer difficulty.

In order to solve the DDSUE problem as a path-based one, we expand the link-based equations for the traffic model by path $p$. Most of the existing path travel time functions assume that the links are independent with each other without considering the influence of bottleneck link. This assumption will lead to the flow on the link being far greater than the link capacity in DTA models. In fact, the bottleneck link will have a serious impact on the smooth traffic operation. Assuming that all the travelers will obtain the traffic information through the traffic information systems in the multimodal transportation network, the congestion of one mode will affect the travel choice in a trip. Therefore, we should determine the real path capacity after obtaining the effective path set. Based on the concept in barrel theory, the capacity of a barrel is determined not by the longest wooden bar or plank, but by the shortest. Therefore, the path capacity is decided by the link which has the minimal capacity on this path. For the path $p=\left\{a_{1}, a_{2}, \ldots, a_{i}, a_{i+1}, \ldots, a_{n}\right\}$, the capacity can be calculated by

$$
Q_{p}^{o d}=\min \left\{Q_{a_{1}}, Q_{a_{2}}, \ldots, Q_{a_{n}}\right\} \quad \forall p, o d,
$$

where $Q_{p}^{o d}$ is the real capacity of the path $p$.
Following the concept of deterministic queue model, the path travel time consists of free travel time and bottleneck travel time. As shown in Figure 3, $a_{2}$ is the bottleneck link and $a_{1}$ and $a_{3}$ are the free flow links; then the path travel time can be calculated by

$$
\tau_{p}(s)=\sum_{a} \Phi_{a} \delta_{a p}+\frac{x_{p}^{o d}(s)}{Q_{p}^{o d}} \quad \forall s,
$$

where $\tau_{p}(s)$ is the travel time on path $p$ given departure at time $s, x_{p}^{o d}(s)$ is the number of vehicles following path $p$ at time $s$, and $Q_{p}^{o d}$ is the real traffic capacity of path $p$.

2.3.3. Flow Propagation Function. In order to describe the dynamic characteristic of traffic flow, the discrete flow propagation function is given by

$$
g_{a}\left(s+\tau_{a}(s)\right)=\frac{e_{a}(s)}{1+\left(\tau_{a}(s)-\tau_{a}(s-1)\right) / \Delta} \quad \forall s .
$$

Under the deterministic queue assumption, the vehicle will move with free-flow speed when the total amount of the inflow into the link $a$ at time $s$ is less than the link capacity $Q_{a}$. The exit flow rate can be expressed as

$$
g_{a}\left(s+\tau_{a}(s)\right)=e_{a}(s) \quad \forall s .
$$

Otherwise, when the total amount of the inflow into the link $a$ at time $s$ is more than the link capacity $Q_{a}$, the vehicles will queue at the exit and the exit flow is equal to the link capacity as

$$
g_{a}\left(s+\tau_{a}(s)\right)=Q_{a} \quad \forall s .
$$

Finally, the formulation to calculate the exit flow can be expressed as

$$
g_{a}(s)= \begin{cases}e_{a}(s)+x_{a}(s-1), & Q_{a}>e_{a}(s)+x_{a}(s-1) \\ Q_{a}, & Q_{a}<e_{a}(s)+x_{a}(s-1) .\end{cases}
$$

The function (16) is expanded to the path level by satisfying the requirements for conservation as

$$
g_{a_{n-1}}(s)=e_{a_{n}}(s),
$$

where link $a_{n}$ is the sequential link that follows link $a_{n-1}$ directly. Moreover, the exit flow and inflow relationship from the path level should be as follows:

$$
g_{p}(s)= \begin{cases}e_{p}(s)+x_{p}(s-1), & Q_{p}>e_{p}(s)+x_{p}(s-1) \\ Q_{p}, & Q_{p}<e_{p}(s)+x_{p}(s-1) .\end{cases}
$$


2.3.4. General Constraints. Except for the above constraints, the DDSUE model should satisfy other general constraints including flow constraints, boundary constraints, and nonnegative constraints, listed as follows:

$$
\begin{gathered}
\left(l_{s}^{o d}\right) \sum_{s=1}^{S} e^{o d}(s)=T^{o d} \quad \forall s, \\
\left(l^{o d}\right) \sum_{p \in P_{o d}} e_{p}(s)=e^{o d}(s), \\
x_{a}(1)=0 \quad \forall a, \\
\left(\lambda_{p}^{o d}\right) e_{p}(s) \geq 0, \quad x_{a}(s) \geq 0 \quad \forall s .
\end{gathered}
$$

\section{Model Formulation}

We assume that each class of travel $i$ has its own value of time (VOT), which is used to calibrate the piecewise linear schedule delay function. Consider three different types of travelers, $i=1,2,3$, namely, the travelers who have high value of time (HVOT), average value of time (AVOT), and low value of time (LVOT). The variational inequality (VI) formulation for the multiclass, multimodal traffic network equilibrium problem can be expressed as follows:

$$
\begin{aligned}
\sum_{i=1}^{3} \sum_{o d \in O D} \sum_{p \in P_{w}^{m}} \sum_{s=1}^{S}\left\{\widehat{c}_{p}(s)\left[e_{p}(s)-e_{p}^{*}(s)\right]\right. \\
+\frac{1}{\theta_{t}} \ln \frac{e^{o d *}(s)}{T^{o d}} \\
\left.\times\left[e^{o d}(s)-e^{o d *}(s)\right]\right\} \geq 0,
\end{aligned}
$$

where $\widehat{c}_{p}(s)=c_{p}\left(e^{o d *}(s), T^{o d}\right)+\left(1 / \theta_{r}\right) \ln \left(e_{p}^{*}(s) / e^{o d *}(s)\right)$, the feasible set is $\Omega=\{9,12,18-22\}$, variables marked with “*” are the solution of the model.

In order to prove the equivalence between (20) and the equilibrium condition of (1) and (7), we derive and analyze the Karush-Kuhn-Tucker conditions of model (20), which are given as

$$
\begin{gathered}
c_{p}(s)+\frac{1}{\theta_{r}} \ln \frac{e_{p}(s)}{e^{o d}(s)}-l_{s}^{o d}-\lambda_{p}^{o d}=0, \\
\frac{1}{\theta_{t}} \ln \frac{e^{o d}(s)}{T^{o d}}+l_{s}^{o d}-l^{o d}=0, \\
e_{p}(s) \lambda_{p}^{o d}=0, \\
\lambda_{p}^{o d} \geq 0 .
\end{gathered}
$$

The form of (21) ensures that $e_{p}(s)>0$. We substitute (23) and (24) into (21) and obtain

$$
\begin{aligned}
\frac{e_{p}(s)}{e^{o d}(s)} & =\exp \left\{\theta_{r}\left[l_{s}^{o d}-c_{p}(s)\right]\right\} \\
& =\exp \left(\theta_{r} l_{s}^{o d}\right) \cdot \exp \left[-\theta_{r} c_{p}(s)\right] .
\end{aligned}
$$

With summation of all the paths and all the kinds of travelers with the consideration of conservation conditions, we can conclude that

$$
\exp \left(\theta_{r} l_{s}^{o d}\right) \sum_{p \in P^{o d}} \exp \left[-\theta_{r} c_{p}(s)\right]=1 .
$$

After substituting (26) into (25), the Logit model (7) can be obtained. Similarly, the Logit model (1) can be derived from (22). Therefore, the proposed VI formulation equation (20) leads to a multiclass, multimodal transportation network equilibrium model where the combined mode-route and departure time choice model assume hierarchical Logit structures.

As the feasible set $\Omega=\{9,12,18-22\}$ consists of a series of linear constraints, therefore the feasible set is a compact convex set. Meanwhile, as $\widehat{c}_{p}(s), \ln \left(e_{p}(s) / e^{o d}(s)\right)$, and $\ln \left(e^{o d}(s) / T^{o d}\right)$ are continuous functions, model (20) exists at least for one solution based on Brouwer fixed-point theorem. Because the path travel time function $\tau_{p}(s)$ does not fit the need of monotony property, it cannot ensure uniqueness of model (20).

\section{Solution Algorithm}

The algorithm based on dynamic stochastic traffic network loading is given to solve the proposed model. The detailed steps can be described as follows.

\section{Step 1. Initialization}

(a) Enumerate all reasonable paths $p \in P_{o d}$ based on reasonable path searching technique [8] and calculate the path capacity based on (11).

(b) Set the value of $e^{\text {od }}\left(s_{1}\right), \Delta$ and $\varepsilon$.

(c) Set an empty network, where $e_{p}^{(0)}(s)=0, \forall p \in$ $P_{o d}, \forall s \in[1, S]$.

(d) Set the iteration number $n=1$ and the start time $s=$ 1 .

Step 2. Dynamic Stochastic Network Loading

(a) Calculate the path travel time $\tau_{p}^{(n)}(s) \forall p \in P_{o d}$ with (12).

(b) Calculate the path travel cost $c_{p}(s), \forall p \in P_{o d}$ with (3).

(c) Compute the minimal expected travel cost $\left\{c^{\text {od(n) }}(s)\right\}$ with (2).

(d) Calculate the departure flow rate $e^{o d(n)}(s)$ with (6) and the path inflow rate with (7).

(e) Compute the path flow using (9) and (16).

(f) If $s<S$, then $s=s+1$ and go to Step 2(a).

Step 3. Convergence Judgment

If $\sum_{o d} \sum_{p \in P_{o d}} \sum_{s=1}^{S}\left|e_{p}^{(n)}(s)-e_{p}^{(n-1)}(s)\right| / \sum_{o d} \sum_{p \in P_{o d}} \sum_{s=1}^{S} e_{p}^{(n)}$ $(s)<\varepsilon$ ( $\varepsilon$ is the convergence criterion), then stop; otherwise $n=n+1$ and $s=1$ and go to Step 2 . 
TABLE 1: Efficient path set.

\begin{tabular}{lcc}
\hline Path & Link & Travel mode \\
\hline 1 & $1-2-3-5-17$ & $a$ \\
2 & $1-2-4-5-17$ & $a$ \\
3 & $1-2-3-4-5-17$ & $a$ \\
4 & $1-2-3-4-6-7-17$ & $c$ \\
5 & $1-2-4-6-7-17$ & $c$ \\
6 & $1-8-9-12-13-17$ & $f$ \\
7 & $1-8-10-6-7-17$ & $e$ \\
8 & $1-11-12-13-17$ & $b$ \\
9 & $1-14-15-16-6-7-17$ & $d$ \\
\hline
\end{tabular}

TABLE 2: Parameters of drive link and transfer link.

\begin{tabular}{lccccc}
\hline Link & $\Phi_{a}$ & $Q_{a}$ & Link & $\Phi_{a}$ & $Q_{a}$ \\
\hline 5 & 200 & 6 & 14 & 380 & 10 \\
6 & 360 & 3 & 15 & 250 & 15 \\
7 & 240 & 4 & 16 & 360 & 12 \\
8 & 300 & 6 & 17 & 40 & 80 \\
9 & 120 & 4 & 18 & 70 & 70 \\
10 & 80 & 50 & 19 & 60 & 100 \\
11 & 500 & 2 & 20 & 40 & 60 \\
12 & 600 & 1 & 21 & 60 & 80 \\
13 & 350 & 12 & & & \\
\hline
\end{tabular}

\section{Numerical Example}

In order to illustrate the performance of the model in this paper, we apply it to two example works, which are a small network and the Sioux Falls network [30]. Through the analysis with these networks, we show that the model produces a DDSUE condition and show the effectiveness of the proposed algorithm.

5.1. A Small Network. First we apply the model to a small network as shown in Figure 1 and link structure as shown in Figure 2. It has 4 traffic modes with $1 O D$ pair. There are six travel modes between the $O D$ pair, where $m=a, b, c$, $d, e, f$ represent the car, bus, car-subway, bus-subway, bicyclesubway, and bicycle-bus travel modes, respectively. Modes $a$ and $b$ are the single-mode travel mode, while the others are the combined travel mode. Nodes 1 and 6 are the origin and destination nodes. Links 1 to 4 and 22 to 24 are the network access/off links, which only express the travel process and do not have the actual travel time. Since the network is relatively small we assume that all feasible paths are used; thus there exists 9 paths for the multimodal network listed in Table 1. Parameters for drive link and transfer link are listed in Table 2.

We suppose that there is no origin-specific cost and that at the destination the unit penalty for early arrival is $m_{1}^{1}=0.5$, $m_{1}^{2}=0.3$, and $m_{1}^{3}=0$, that for the late arrival is $m_{2}^{1}=1, m_{2}^{2}=$ 0.8 , and $m_{2}^{3}=0.5$, that the ideal arrival time is $\bar{s}=600 \mathrm{~s}$, and that the tolerance on the desired arrival time is $\delta=100 \mathrm{~s}$. We take the initial departure rate $e^{o d}(1)=0.1$ trips/second and

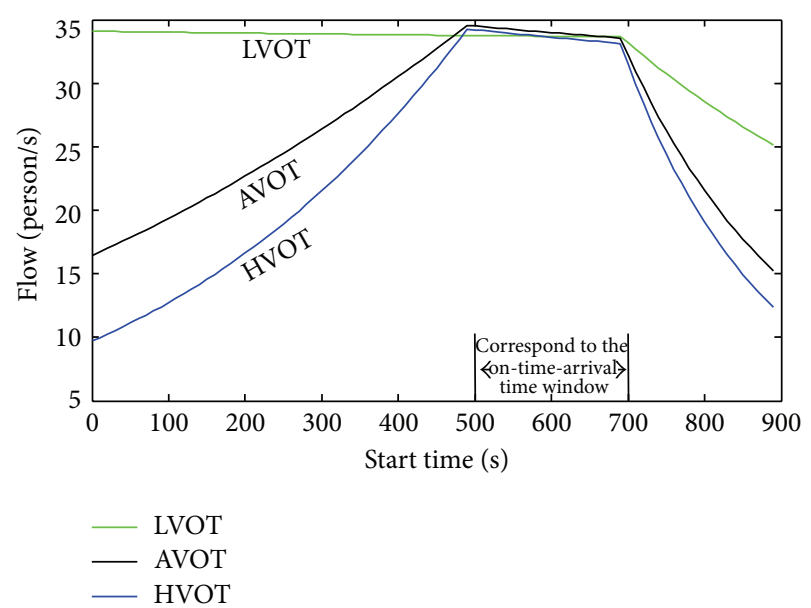

FIGURE 4: Departure flow for the $O D$ pair with multiclass users.

the time increment $\Delta=10 \mathrm{~s}$. Other parameters are as follows: $\varepsilon=0.01, \theta_{t}=0.005$, and $\theta_{r}=0.01$.

After about 27 iterations, the program can reach the convergence precision in 0.86 seconds. The departure flows calculated from the algorithm are shown in Figure 4, while the costs incurred on the $O D$ pair are shown in Figure 5. These two figures show that the LVOT travelers would begin their trips early due to minimal penalty for early arrival at the destination, while the trips of LVOT travelers decrease after the on-time-arrival time window. The cost for LVOT travelers also maintains a certain level and increases after the on-timearrival time window. Most HVOT travelers would begin their trips so as to arrive at the destination within the on-timearrival time window because the penalties for being early and late are both expensive. The departures for AVOT travelers are somewhere in between.

For in-depth research, we discuss the results for different modal paths by considering only one class of travelers: AVOT travelers in the network. The departure flow for all the paths in the $O D$ pair is shown in Figure 6. It can be observed that the function curve trends for different paths of departure time are the same; most of the travelers choose to leave in order to arrive within the on-time-arrival time window. Due to the same cost of paths 1 and 2 and paths 3 and 4, the departure flow curves coincide. In general, the travelers who choose the car travel mode (paths 1,2, and 3) are the most numerous as its travel cost (in term of travel time) is the lowest. Subway trips are more attractive than bus trips due to the punctuality and low cost. The departure flow of the car trips among all travel modes is the highest, followed by car-subway, bicycle-subway, bus-subway, bus, and bicycle-bus.

The departure cost for all the paths in the $O D$ pair is shown in Figure 7. There exists strong relationship between Figures 6 and 7. The departure cost for all travel modes decreases at first then remains stable to a certain degree and gradually increases. It is because that traveler will be enduring the penalty by starting early due to the penalty cost function; the cost becomes increasingly smaller and remains at the minimum cost as the departure time approaches to ideal 


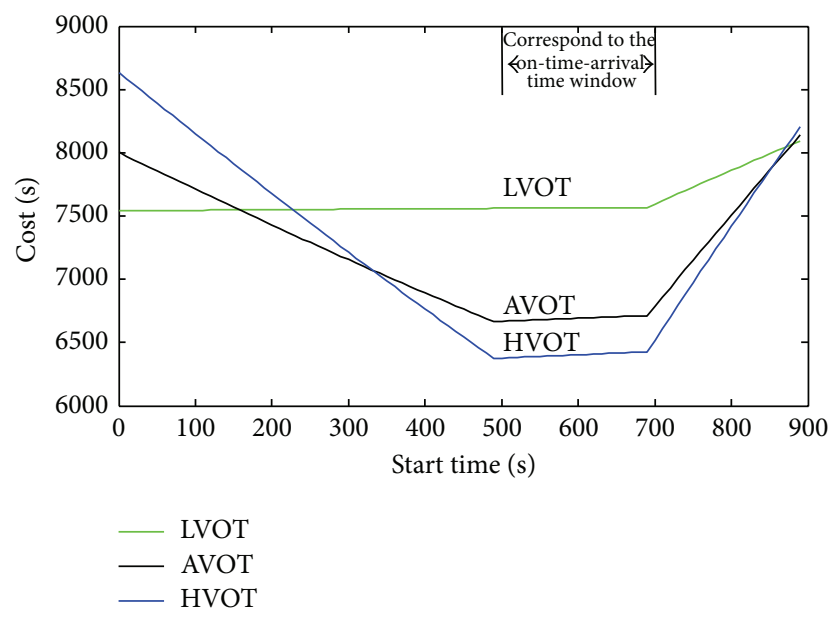

Figure 5: Departure cost for the $O D$ pair with multiclass users.

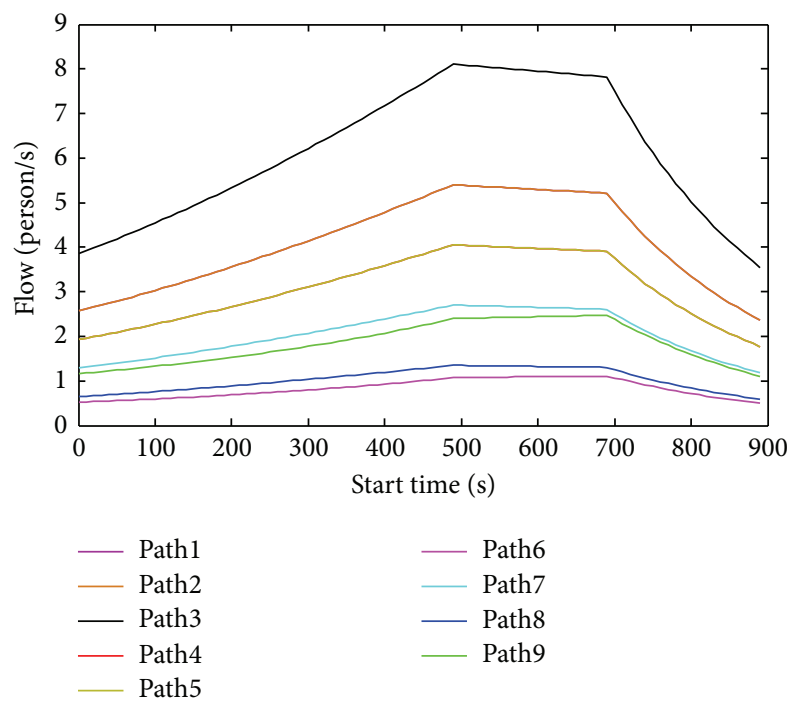

FIgURE 6: Departure flow for the $O D$ pair.

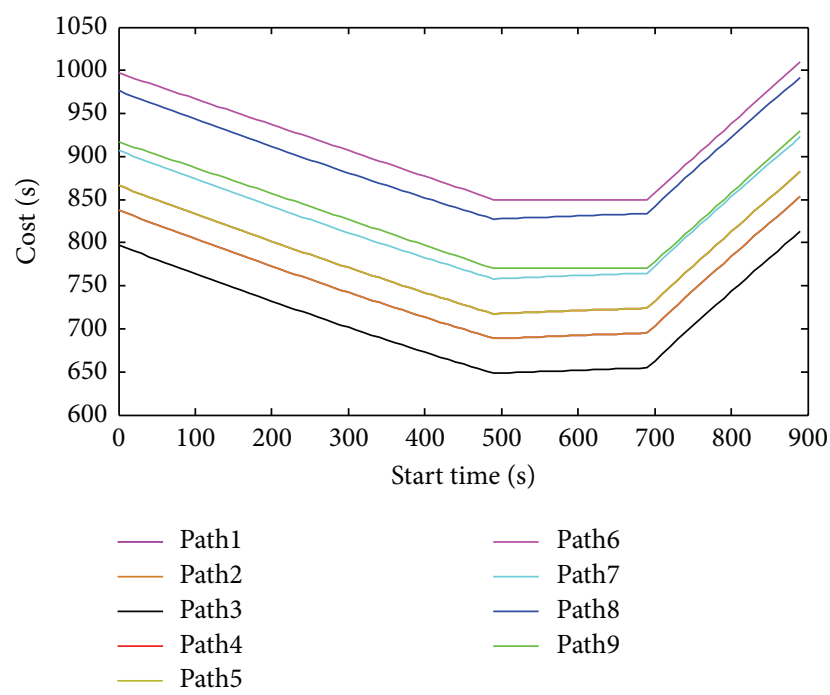

FIgURE 7: Departure cost for the OD pair.

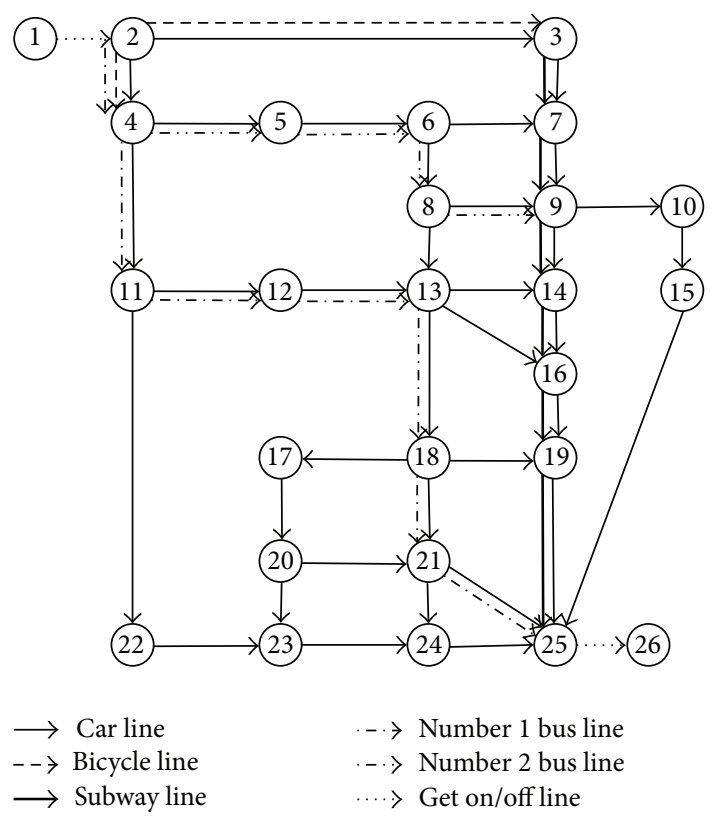

FIGURE 8: Normal medium-sized road network.

arrival time; travelers will also be penalized by the late departure time; the later they depart, the greater is the cost. As the penalty coefficient of late arrival is larger than the one of early arrival, the travel cost for early arrival is lesser than late arrival for the same length of time deviation. For example, the travel cost starting at $300 \mathrm{~s}$ is lesser than at $900 \mathrm{~s}$, even though both of them have the same $300 \mathrm{~s}$ time difference with respect to the ideal arrival time $600 \mathrm{~s}$.

5.2. The Sioux Fall Network. The second test is performed with the Sioux Fall network [30], which has one OD pair (1, 26) and four traffic modes (car, bicycle, subway, and bus), 1 subway line, and 2 bus lines, as shown in Figure 8. Based on the method in Section 2, the corresponding super network is given in Figure 9. Nodes 1 and 48 are the origin and destination nodes. Links 1 to 3 and 66 to 68 are the network access/off links, which only express the travel process and do not have actual travel time.

Parameters for drive link and transfer link are listed in Table 3. Parameters are given as $\left[s_{0}, s_{1}\right]=[0,495], \Delta=5 \mathrm{~s}, \bar{s}=$ 350 , and $\delta=50$, while the other parameters are the same with the previous experiment.

After about 34 iterations (see Figure 10), the program can reach the convergence precision in 6.5 seconds and provides 54 effective paths. The experiment results demonstrate the algorithm's effectiveness in solving the DDSUE problem, which can be applied in the medium and large networks.

\section{Conclusions}

In this paper, we have identified a new multiclass, multimodal equilibrium condition for dynamic departure time and stochastic user equilibrium (DDSUE), together with a model formulation and solution algorithm. Three classes of travelers 
TABLE 3: Parameters of driving link and transfer link.

\begin{tabular}{lcccccccccccccc}
\hline Link & $\Phi_{a}$ & $Q_{a}$ & Link & $\Phi_{a}$ & $Q_{a}$ & Link & $\Phi_{a}$ & $Q_{a}$ & Link & $\Phi_{a}$ & $Q_{a}$ & Link & $\Phi_{a}$ & $Q_{a}$ \\
\hline 4 & 210 & 5 & 17 & 85 & 4 & 30 & 120 & 3 & 43 & 40 & 25 & 56 & 90 & 7 \\
5 & 75 & 5 & 18 & 70 & 4 & 31 & 75 & 3 & 44 & 90 & 25 & 57 & 160 & 8 \\
6 & 70 & 4 & 19 & 280 & 5 & 32 & 70 & 5 & 45 & 300 & 1 & 58 & 25 & 40 \\
7 & 80 & 4 & 20 & 150 & 4 & 33 & 85 & 6 & 46 & 250 & 1 & 59 & 20 & 35 \\
8 & 70 & 4 & 21 & 120 & 3 & 34 & 70 & 6 & 47 & 65 & 6 & 60 & 25 & 40 \\
9 & 65 & 5 & 22 & 80 & 5 & 35 & 75 & 6 & 48 & 70 & 5 & 61 & 20 & 30 \\
10 & 155 & 5 & 23 & 80 & 5 & 36 & 85 & 3 & 49 & 75 & 6 & 62 & 20 & 50 \\
11 & 85 & 3 & 24 & 90 & 4 & 37 & 70 & 3 & 50 & 80 & 6 & 63 & 30 \\
12 & 80 & 5 & 25 & 75 & 4 & 38 & 320 & 3 & 51 & 65 & 5 & 64 & 25 \\
13 & 65 & 4 & 26 & 70 & 3 & 39 & 45 & 25 & 52 & 150 & 6 & 65 & 30 \\
14 & 80 & 3 & 27 & 80 & 4 & 40 & 50 & 25 & 53 & 85 & 6 & 40 \\
15 & 70 & 5 & 28 & 150 & 5 & 41 & 50 & 25 & 54 & 80 & 6 & &
\end{tabular}

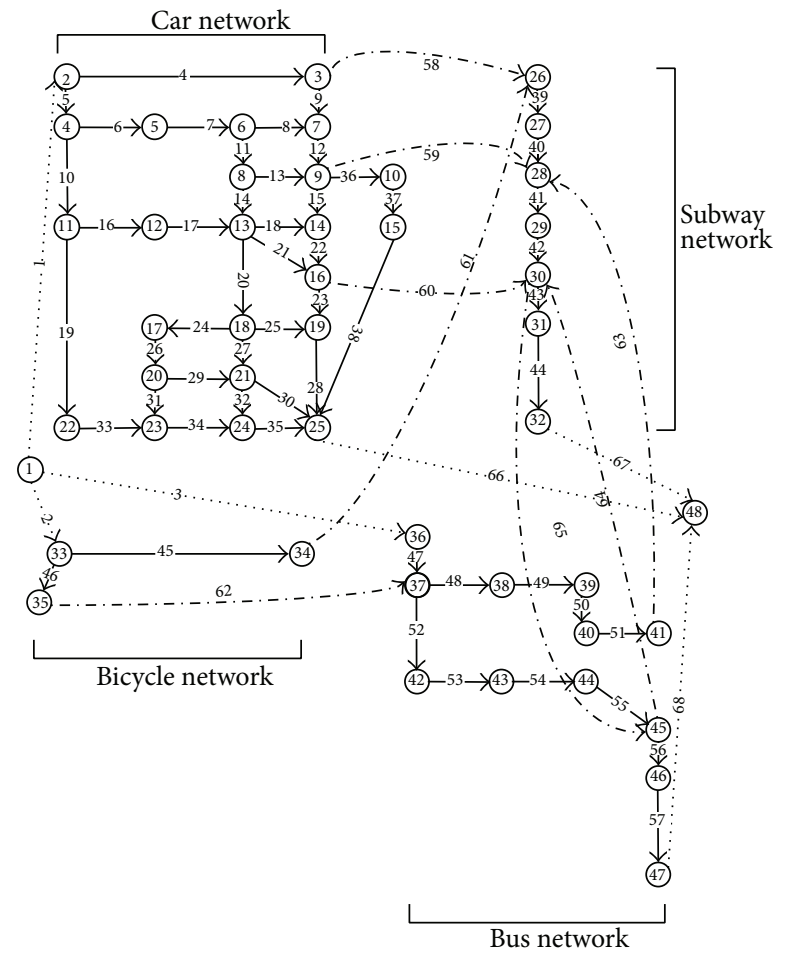

$\longrightarrow$ Running line

$\rightarrow$ Transfer line

Figure 9: Super network.

with different values of time are considered in the multimodal transportation network. All classes of travelers choose not only the travel route, but also the traffic modes and the departure time. The bottleneck restriction in the multimodal network is taken into account to ensure the maximum use of the network capacity. The network equilibrium condition is analyzed by the Logit structure considering the time dependent travel cost and schedule delay. The equivalent variational

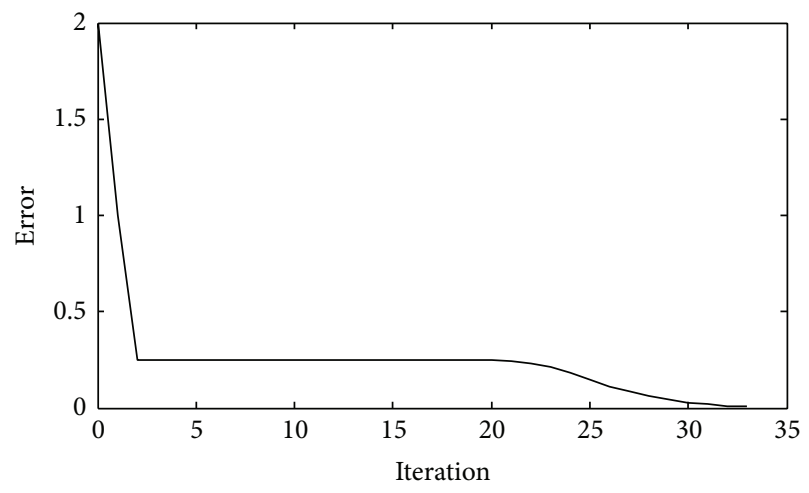

FIGURE 10: Convergence of the proposed algorithm.

inequality model is established and solved by an algorithm based on dynamic stochastic network loading.

Results from numerical experiments show a continuous monotonic decreasing relation between departure flow and departure cost, which implies the existence of equilibrium. Travelers with different values of time (VOT) have different requirements for the departure time. Travelers with low value of time (LVOT) would prefer to set the departure ahead as they have minimal penalty for early arrival; conversely, travelers with high value of time (HVOT) would start their trip on time to avoid the early and late penalties. The departures for average value of time (AVOT) travelers are somewhere in between. Moreover, the application to the Sioux Falls network shows that in a general transportation network, the model and solution algorithm converges well within a reasonable computing time.

\section{Conflict of Interests}

The authors declare that there is no conflict of interests regarding the publication of this paper. 


\section{Acknowledgments}

The work described in this paper is supported by the National Natural Science Foundation of China (51178032), International Cooperation and Exchange of the National Natural Science Foundation of China (no. 71210001), and Nanyang Technological University Sustainable Earth Office (Project: Modelling Multimodal Travel Behaviour Linking Nonmotorised Transport with Public Transport).

\section{References}

[1] D. K. Merchant and G. L. Nemhauser, "A model and an algorithm for the dynamic traffic assignment," Transportation Science, vol. 12, no. 3, pp. 62-77, 1976.

[2] S. Peeta and A. K. Ziliaskopoulos, "Foundations of dynamic traffic assignment: the past, the present and the future," Networks and Spatial Economics, vol. 1, no. 3-4, pp. 233-265, 2001.

[3] Q. Meng and H. L. Khoo, "A computational model for the probit-based dynamic stochastic user optimal traffic assignment problem," Journal of Advanced Transportation, vol. 46, no. 1, pp. 80-94, 2012.

[4] S. Gao, "Modeling strategic route choice and real-time information impacts in stochastic and time-dependent networks," IEEE Transactions on Intelligent Transportation Systems, vol. 13, no. 3, pp. 1298-1311, 2012.

[5] B. Fu, C. Zhao, and S. Li, "The analysis of Braess' paradox and robustness based on dynamic traffic assignment models," Discrete Dynamics in Nature and Society, vol. 2013, Article ID 796842, 8 pages, 2013.

[6] H.-K. Chen and M.-S. Chang, "Dynamic user-optimal departure time/route choice with hard time-windows," Journal of Transportation Engineering, vol. 126, no. 5, pp. 413-418, 2000.

[7] H. Huang and W. H. K. Lam, "Modeling and solving the dynamic user equilibrium route and departure time choice problem in network with queues," Transportation Research Part B: Methodological, vol. 36, no. 3, pp. 253-273, 2002.

[8] Y. Lim and B. Heydecker, "Dynamic departure time and stochastic user equilibrium assignment," Transportation Research Part B: Methodological, vol. 39, no. 2, pp. 97-118, 2005.

[9] S. Lee, "Multimodal, multiclass stochastic dynamic traffic assignment for evaluating information provision strategies," Journal of Advanced Transportation, vol. 42, no. 1, pp. 45-64, 2008.

[10] J. S. Mun, "A model and solution algorithm for dynamic deterministic user equilibrium assignment," Transportation Planning and Technology, vol. 32, no. 6, pp. 461-497, 2009.

[11] A. K. Ziliaskopoulos and L. Rao, "A simultaneous route and departure time choice equilibrium model on dynamic networks," International Transactions in Operational Research, vol. 6, no. 1, pp. 21-37, 1999.

[12] W. Y. Szeto and H. K. Lo, "A cell-based simultaneous route and departure time choice model with elastic demand," Transportation Research Part B: Methodological, vol. 38, no. 7, pp. 593-612, 2004.

[13] C. Hendrickson and G. Kocur, "Schedule delay and departure time decision in a deterministic model," Transportation Science, vol. 15, no. 1, pp. 62-77, 1981.

[14] B. Ran and D. E. Boyce, Modelling Dynamic Transportation Network: An Intelligent Transportation System Oriented Approach, Springer, Berlin, Germany, 1996.
[15] H. K. Chen, Dynamic Travel Choice Models, Springer, Berlin, Germany, 1999.

[16] W. H. K. Lam and H. Huang, "A combined activity/travel choice model for congested road networks with queues," Transportation, vol. 29, no. 1, pp. 3-29, 2002.

[17] W. H. K. Lam, Z. Li, H. Huang, and S. C. Wong, "Modeling timedependent travel choice problems in road networks with multiple user classes and multiple parking facilities," Transportation Research Part B: Methodological, vol. 40, no. 5, pp. 368-395, 2006.

[18] S. Li, "Genetic algorithm for solving dynamic simultaneous route and departure time equilibrium problem," Transport, vol. 23, no. 1, pp. 73-77, 2008.

[19] M. Meng, C. F. Shao, J. J. Zeng, X. X. Lin, and X. Ji, "Dynamic route choice model with departure time in a combined trip," Journal of Central South University of Technology. In press.

[20] M. Tagmouti, M. Gendreau, and J. Potvin, "A dynamic capacitated arc routing problem with time-dependent service costs," Transportation Research C: Emerging Technologies, vol. 19, no. 1, pp. 20-28, 2011.

[21] W. X. Wu and H. J. Huang, "A path-based gradient projection algorithm for the cost-based system optimum problem in networks with continuously distributed value of time," Journal of Applied Mathematics, vol. 2014, Article ID 271358, 9 pages, 2014.

[22] M. E. Ben-Akiva, S. Gao, Z. Wei, and Y. Wen, "A dynamic traffic assignment model for highly congested urban networks," Transportation Research Part C: Emerging Technologies, vol. 24, pp. 62-82, 2012.

[23] Z. Qu, Y. Xing, X. Song, Y. Duan, and F. Wei, "A study on the coordination of urban traffic control and traffic assignment," Discrete Dynamics in Nature and Society, vol. 2012, Article ID 367468, 12 pages, 2012.

[24] C. C. Lu, H. S. Mahmassani, and X. S. Zhou, "A bi-criterion dynamic user equilibrium traffic assignment model and solution algorithm for evaluating dynamic road pricing strategies," Transportation Research Part C: Emerging Technologies, vol. 16, no. 4, pp. 371-389, 2008.

[25] X. Zhang, H. Yang, and H. Huang, "Multiclass multicriteria mixed equilibrium on networks and uniform link tolls for system optimum," European Journal of Operational Research, vol. 189, no. 1, pp. 146-158, 2008.

[26] X. Wang and H. Huang, "Bi-criteria system optimum traffic assignment in networks with continuous value of time," PROMET-TrafficeTransportation, vol. 25, no. 2, pp. 119-125, 2013.

[27] Z. X. Wu and W. H. K. Lam, "Network equilibrium for congested multi-mode networks with elastic demand," Journal of Advanced Transportation, vol. 37, no. 3, pp. 295-318, 2003.

[28] W. S. Vickrey, "Congestion theory and transport investment," The American Economics Review, vol. 59, pp. 251-261, 1969.

[29] R. Arnott, A. de Palma, and R. Lindsey, "Departure time and route choice for the morning commute," Transportation Research B. Methodological, vol. 24, no. 3, pp. 209-228, 1990.

[30] L. J. Leblanc, "An algorithm for the discrete network design problem," Transportation Science, vol. 9, no. 3, pp. 183-199, 1975. 


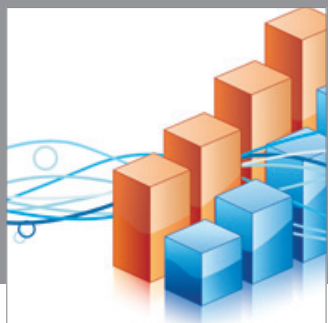

Advances in

Operations Research

mansans

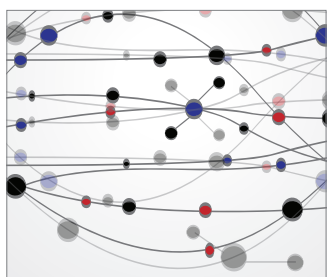

The Scientific World Journal
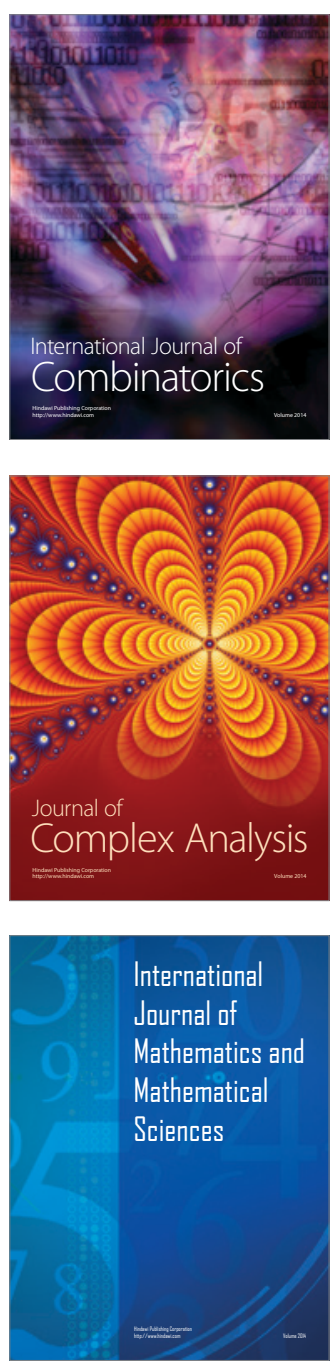
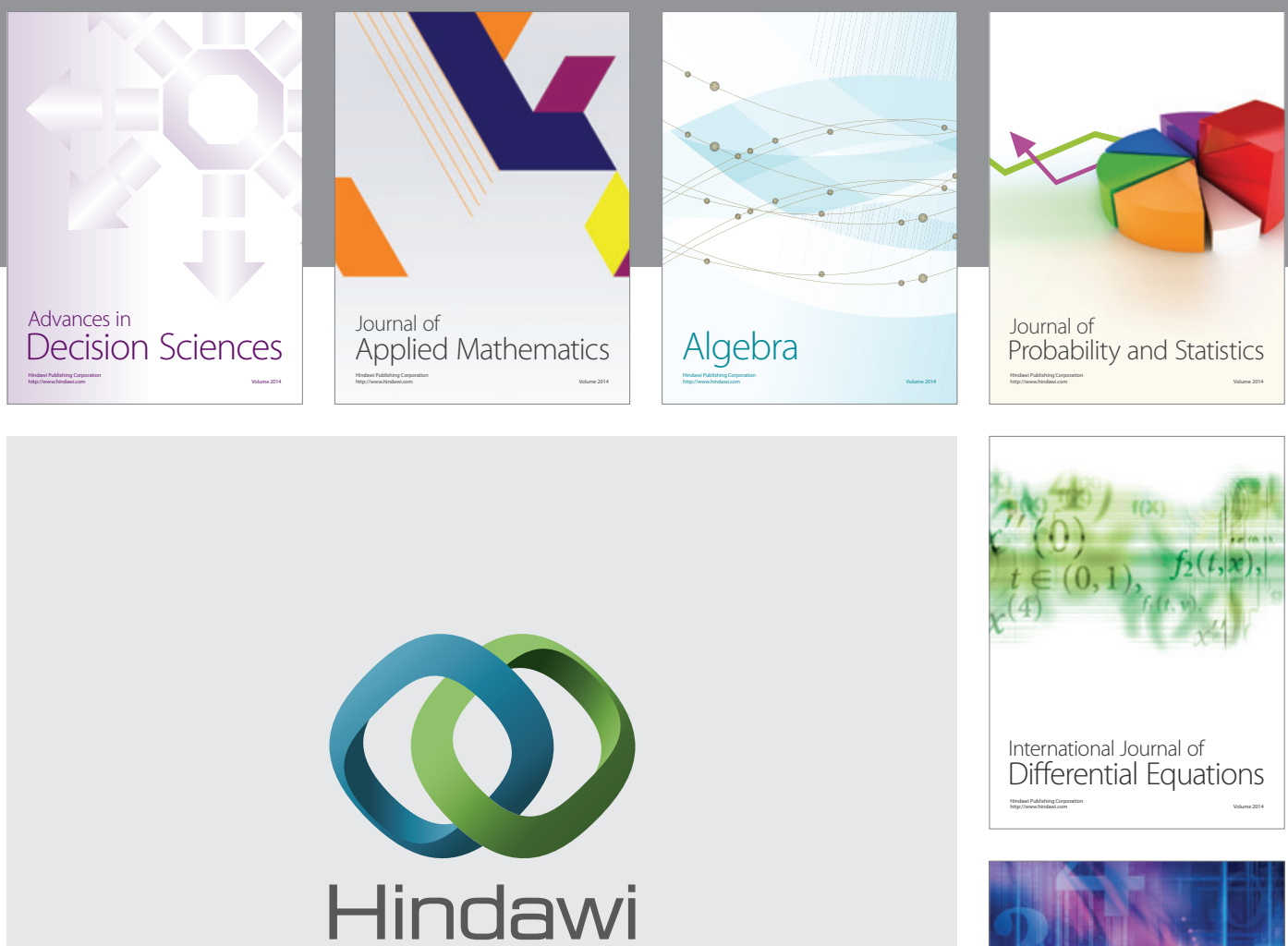

Submit your manuscripts at http://www.hindawi.com
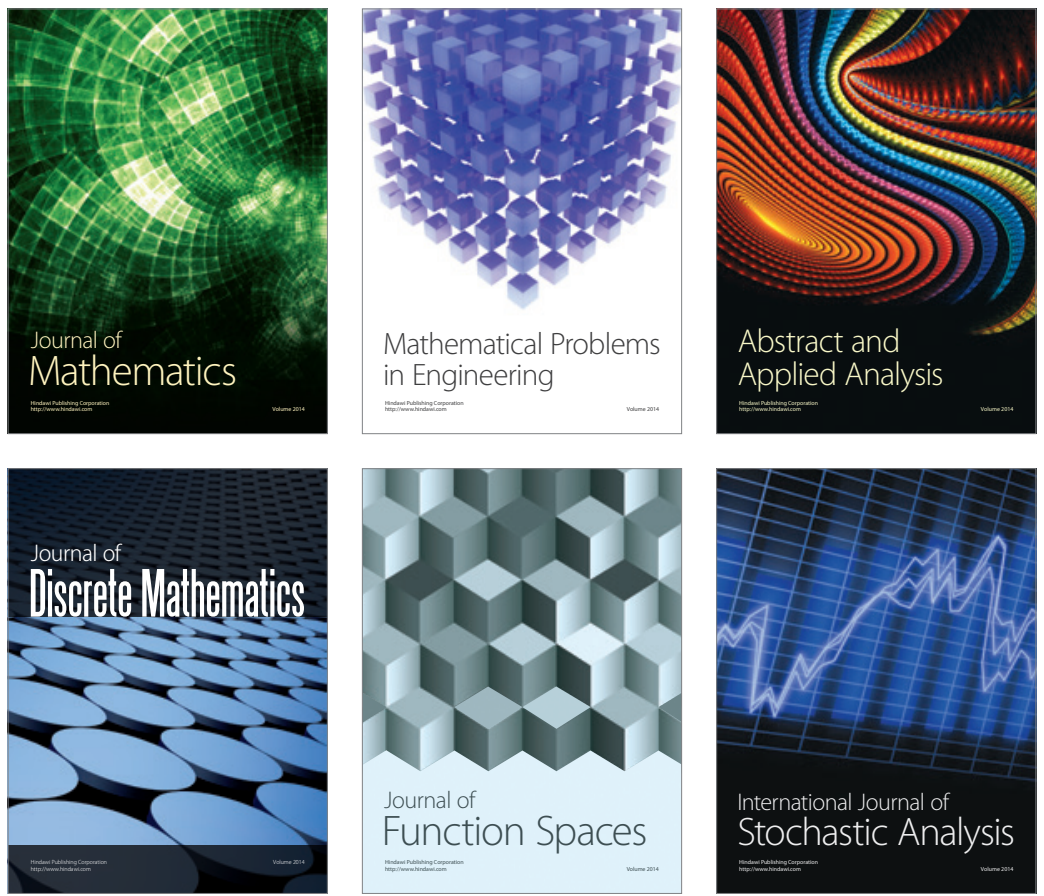

Journal of

Function Spaces

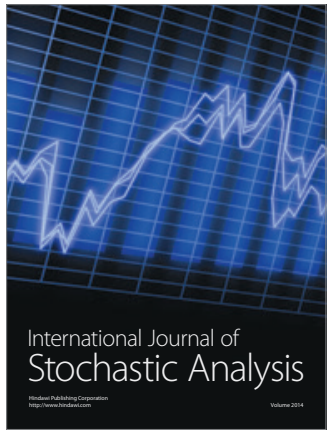

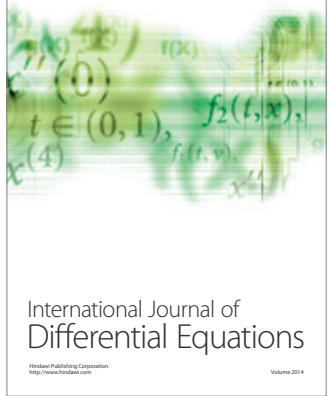
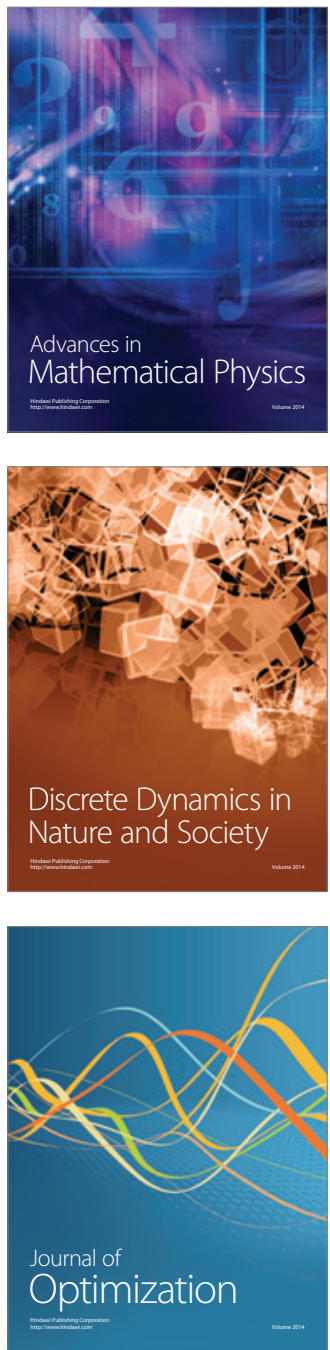